\title{
A parallel in time approach for quantum control: the parareal algorithm
}

\author{
Yvon Maday \\ Laboratoire Jacques-Louis Lions \\ Université Pierre et Marie Curie \\ Boîte courrier 187, 75252 Paris Cedex 05 France \\ maday@ann . jussieu.fr \\ Gabriel TURINICI \\ INRIA Rocquencourt, Domaine de Voluceau \\ Rocquencourt - B.P. 105, 78153 \\ Le Chesnay Cedex - France \\ and \\ CERMICS-ENPC, Marne la Vallée, France \\ Gabriel.Turinici@inria.fr
}

\begin{abstract}
The numerical studies of control problems in quantum chemistry go through the computer simulation of the dynamical phenomena involved. These simulations are in many cases too expensive to be carried out for complex systems, precluding thus treatment of interesting practical situations.

In a context of fast increasing in both the CPU power available on typical workstations and of the number of computers that can be connected through high speed networks, the difficulty lies rather in how to obtain "real time" solutions than in the amount of CPU power available (which becomes to exceed the needs).

In this context, the "parareal" time algorithm that parallelize in the time direction the effort required to solve evolution equations has been introduced in previous works $[4,3,2]$. The theoretical modifications required in or-
\end{abstract}

der to apply this algorithm to quantum control problems together with numerical evidence is the main topic of this paper.

\section{Introduction}

The control of quantum mechanical systems has long tradition in using numerical computer simulations in order to model and understand intricate practical situations encountered in complex laboratory experiments [1]. However, even when these simulations refer to simple systems they are very costly in computational resources and require long times to be carried on. On the other hand, in the nowadays hardware environments, CPU power is not such a scarce resource and, for instance, the price of constructing a high-speed networked cluster of PCs is now within the financial means of most academic departments. The bottleneck comes thus not from the lack of processors but rather from the intrinsic sequential nature of 
the simulations used in quantum control which mostly deal with evolution equations. In order to address this situation, a discretization scheme parallel in time was proposed [4]; this scheme, called the "parareal" time scheme, decomposes the time evolution in several independent sub-problems which are evolved simultaneously (in parallel) on different processors. Necessary corrections are then applied to the results thus obtained in an iterative manner leading to (fast) convergence to the exact result (allowing to envision real time simulations). This scheme was applied with success to the linear [4] and non-linear PDEs [3], to the equations of the molecular dynamics [2] and also for a first application to control problems [5]. Within this context, the goal of this paper is to discuss the specificities required by the application of this "parareal" time scheme to quantum control settings. The necessary dynamical equations are presented in Section 2 together with the adequated algorithms to solve the control problem. The parareal scheme is presented in Section 3 followed in Section 4 by implementation details and numerical results. Finally, a discussion and some perspectives are developped in Section 5.

\section{Dynamical Equations}

Consider a quantum system with internal Hamiltonian $H_{0}$ without control interaction that is prepared in the initial state $\psi_{0}(x)$ (with $\left.\left\|\psi_{0}\right\|_{L^{2}\left(\mathbf{R}^{\gamma}\right)}=1\right)$ where $x$ denotes the relevant coordinate variables; the state $\psi(x, t)$ of the system satisfies at each time $t$ the timedependent Schrödinger equation

$$
\left\{\begin{array}{l}
i \hbar \frac{\partial}{\partial t} \psi(x, t)=H_{0} \psi(x, t) \\
\psi(x, t=0)=\psi_{0}(x)
\end{array}\right.
$$

In the presence of an external interaction taken here as an electric field modeled by a coupling operator given by an amplitude $\epsilon(t) \in \mathbf{R}$ and a time independent dipole moment operator $\mu$ the Hamiltonian $H_{0}$ is replaced by $H=H_{0}-$ $\epsilon(t) \mu$ that gives rise to the dynamical equations to be controlled:

$$
\left\{\begin{array}{l}
i \hbar \frac{\partial}{\partial t} \psi(x, t)=\left(H_{0}-\epsilon(t) \mu\right) \psi(x, t) \\
\psi(x, t=0)=\psi_{0}(x)
\end{array}\right.
$$

The optimal control approach is to assess the fitness of the final state $\psi(T)=\psi(x, T)$ through the introduction of a cost functional $J$ to be optimized; this cost functional includes on one hand terms that describe how well the objective has been met and on the other hand terms that penalize undesired effects. One simple example of cost functional is

$$
J(\epsilon)=<\psi(T)|O| \psi(T)>-\alpha \int_{0}^{T} \epsilon^{2}(t) d t
$$

where $\alpha>0$ is a parameter and $O$ is the observable operator that encodes the goal. In mathematical terms, the observable $O$ is a selfadjoint operator that acts on $\psi(T)$; larger the value $<\psi(T)|O| \psi(T)>$ better the control objectives have been met; note that in general attaining the maximal possible value of $<\psi(T)|O| \psi(T)>$ requires a large laser fluence $\int_{0}^{T} \epsilon^{2}(t) d t$; the optimum evolution will therefore strike a balance between using a not too expensive laser fluence while simultaneously ensuring the desired observable has a sufficiently large value.

The maximization of the cost functional $J(\epsilon)$ can be realized by writing down the EulerLagrange critical point equations; a standard way to derive these equations is to introduce an adjoint state $\chi(t, x)$ (used as a Lagrange multiplier). The following equations are thus obtained [9]:

$$
\begin{aligned}
& \left\{\begin{array}{l}
i \hbar \frac{\partial}{\partial t} \psi(x, t)=\left(H_{0}-\epsilon(t) \mu\right) \psi(x, t) \\
\psi(x, t=0)=\psi_{0}(x)
\end{array}\right. \\
& \left\{\begin{array}{l}
i \hbar \frac{\partial}{\partial t} \chi(x, t)=\left(H_{0}-\epsilon(t) \mu\right) \chi(x, t) \\
\chi(x, t=T)=O \psi(x, T)
\end{array}\right. \\
& \alpha \epsilon(t)=-\operatorname{Im}<\chi(t)|\mu| \psi(t)>
\end{aligned}
$$

One efficient choice for solving in practice the critical point equations (4)-(6) is the 
monotonically convergent iterative algorithm of Zhu\&Rabitz [9] that can be described by the resolution of the following equations at step $k$ :

$\left\{\begin{array}{l}i \hbar \frac{\partial}{\partial t} \psi^{k}(x, t)=\left(H_{0}+\frac{\operatorname{Im}<\chi^{k-1}|\mu| \psi^{k}>(t)}{\alpha} \mu\right) \psi^{k}(x, t) \\ \psi^{k}(x, t=0)=\psi_{0}(x)\end{array}\right.$

$\left\{\begin{array}{l}i \hbar \frac{\partial}{\partial t} \chi^{k}(x, t)=\left(H_{0}+\frac{\operatorname{Im}<\chi^{k}|\mu| \psi^{k}>(t)}{\alpha} \mu\right) \chi^{k}(x, t) \\ \chi^{k}(x, t=T)=O \psi^{k}(x, T)\end{array}\right.$

Let us also mention the formulation of [6] that was also used here

$$
\begin{aligned}
& \left\{\begin{array}{l}
i \hbar \frac{\partial}{\partial t} \psi^{k}(x, t)=\left(H_{0}-\epsilon^{k}(t) \mu\right) \psi^{k}(x, t) \\
\psi^{k}(x, t=0)=\psi_{0}(x)
\end{array}\right. \\
& \epsilon^{k}=(1-\delta) \tilde{\epsilon}^{k-1}-\frac{\delta}{\alpha} \operatorname{Im}\left\langle\chi^{k-1}|\mu| \psi^{k}\right\rangle \\
& \left\{\begin{array}{l}
i \hbar \frac{\partial}{\partial t} \chi^{k}(x, t)=\left(H_{0}-\tilde{\epsilon}^{k}(t) \mu\right) \chi^{k}(x, t) \\
\chi^{k}(x, t=T)=O \psi^{k}(x, T)
\end{array}\right. \\
& \tilde{\epsilon}^{k}=(1-\eta) \epsilon^{k}-\frac{\eta}{\alpha} \operatorname{Im}\left\langle\chi^{k}|\mu| \psi^{k}\right\rangle
\end{aligned}
$$

\section{Parareal time discretization}

Consider a general evolution equation

$$
\frac{d}{d t} u(t)=A u(t)
$$

and define the associated propagator $F\left(t_{0}, t_{1}\right)$ by the requirement that for any $u, F\left(t_{0}, t_{1}\right) u$ is the solution at time $t_{1}$ of Eqn. (13) for the initial data $u$ at time $t_{0}$. Given a final time $T$ and an initial data $u_{0}$, solving Eqn. (13) reduces to computing $U(t)=F(0, t) u, 0 \leq t \leq T$. Let us introduce intermediary times $T_{n}=n \cdot T / N$, $n=0, . ., N$ and intermediate time state snapshots $u_{n}, n=1, \ldots, N$. It is obvious that $u_{n}$ are exactly the values $U\left(T_{n}\right)$ if and only if for any $n=1, \ldots, N: u_{n}=F\left(T_{n-1}, T_{n}\right) u_{n-1}$.

The parareal time scheme proposes to give an iterative method to obtain the sequence $U\left(T_{n}\right), n=1, \ldots, N$ using a coarse propagator $G\left(T_{n-1}, T_{n}\right)$ that approximates $F\left(T_{n-1}, T_{n}\right)$ $(n=1, \ldots, N)$ obtained e.g. by solving a cheap, approximate, yet close to original evolution equation

$$
\frac{d}{d t} u(t)=\tilde{A} u(t)
$$

The iterative update procedure is then

$$
\left\{\begin{array}{l}
u_{0}^{k+1}=u_{0} \\
u_{n+1}^{k+1}=G\left(T_{n}, T_{n+1}\right) u_{n}^{k+1}+ \\
F\left(T_{n}, T_{n+1}\right) u_{n}^{k}-G\left(T_{n}, T_{n+1}\right) u_{n}^{k}
\end{array}\right.
$$

In this scheme, for each iteration $k$ the computation of $F\left(T_{n}, T_{n+1}\right) u_{n}^{k}-G\left(T_{n}, T_{n+1}\right) u_{n}^{k}(n=$ $1, \ldots, N)$ is to be done in parallel and the only sequential part remains the computation of $G\left(T_{n}, T_{n+1}\right) u_{n}^{k+1}$ which is cheap since it involves the coarse propagator.

When this scheme is applied to the setting of the quantum control, a desirable property is that the update formula of Eqn. (15) be consistent with the norm preservation properties of the evolution equation (4): $\|\psi(x, t)\|=$ $\left\|\psi_{0}(x)\right\|=1, \forall t$. In order to address this issue an alternative update scheme will also be used in this work:

$$
u_{0}^{k+1}=u_{0}, u_{n+1}^{k+1}=\mathcal{R}_{n} F\left(T_{n}, T_{n+1}\right) u_{n}^{k}
$$

where $\mathcal{R}_{n}$ is the rotation in the plane spanned by $G\left(T_{n}, T_{n+1}\right) u_{n}^{k} \quad$ and $\quad G\left(T_{n}, T_{n+1}\right) u_{n}^{k+1}$ that transforms $G\left(T_{n}, T_{n+1}\right) u_{n}^{k}$ into $G\left(T_{n}, T_{n+1}\right) u_{n}^{k+1}$. This update formula will preserve the norm of the state as soon as the fine evolution $F\left(T_{n}, T_{n+1}\right)$ is norm preserving.

\section{Choice of coarse propagator and numerical results}

In order to test the parareal algorithm for quantum control problems we have chosen the case of a Morse potential type $O-H$ bond. The model was taken to be the one described in [8] page 1958 (first test case: from $\psi_{0}=|0\rangle$ to $\psi_{T}=|1\rangle, T=30000$ a.u.). The fine propagator $F$ was taken to correspond to the splitoperator propagation method (of second order in the state and first-order in the field) 
with a time step $d t=1$ a.u. and a spacial discretization of $d x=0.234$ a.u. The coarse propagator $G$ was taken to correspond to the same numerical scheme but with a larger time step, $D T=T / N=10$ a.u.; it is therefore $D T / d t=10$ times faster to apply the coarse propagator $G$ than it is to compute the fine propagator $F$.

Remark. One can also think of a coarse propagator that corresponds to the evolution of the system within the space spanned by the first $\ell$ eigenfunctions of the internal Hamiltonian $H_{0}$ (e.g. $\ell=10)$. The numerical results corresponding to this approach, not reported here, show promising perspectives but work is still needed in this direction to improve its performance.

In the first test of our parallel scheme we consider that the optimal field $\epsilon(t)$ that maximizes $J(\epsilon)$ is known and we check that the parareal scheme is able to recover the evolution of the system. The numerical results are presented in Fig. 1 and 2 where it is demonstrated that the parareal scheme improves the results obtained by the coarse propagator only. Depending on the tolerance, two or three parareal iterations suffice, which results in a speed-up by a factor of 5 or 3.33 respectively. As expected, the performance of the scheme in Eqn. (16) is better than that of Eqn. (15).

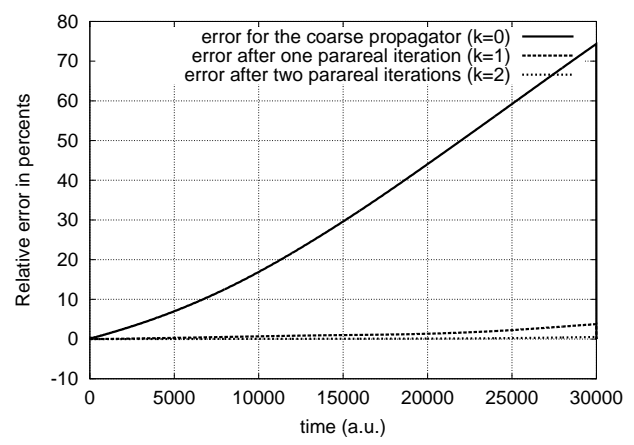

Figure 1: Result of the parareal rotation scheme in Eqn. (16). Negligible error is recovered after only 3 parareal iterations.

Finally, the quantum control problem was considered. The problem was solved with the

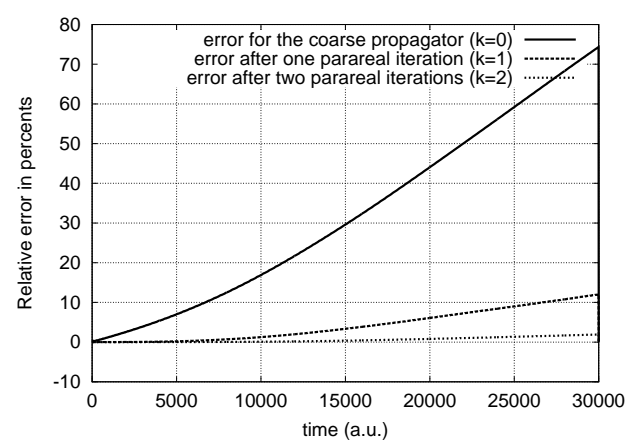

Figure 2: Result of the parareal linear scheme in Eqn. (15). Small error is recovered after only 3 parareal iterations. It is seen that the error decay is slower than that of the scheme in Eqn. (16) and Fig. 1.

monotonic convergent algorithm in Eqns (9)(11) (with $\delta=0.25=\eta, \delta=1=\eta$ and $\delta=2, \eta=0$ ) in each (nonlinear) propagation the parareal scheme was employed, with only one parareal iteration per evolution equation. The results in Fig $3(\delta=1=\eta)$ show that a good overlap with the objective operator is reached Our actual implementation is not yet parallel, but we estimate that a parallel implementation would yield in an overall gain factor (in real time required to solve the problem) of the order of 5 .

For this case other choices for the parameters $\delta$ and $\eta$ give the expected behavior [6]: for instance $\delta=0.25=\eta$ converges slower than $\delta=1.0=\eta$ that is turn converges slower than $\delta=2, \eta=0$. For other cases not reported here we could identify some choices of time steps for which the setting $\delta=0.25=\eta$ converges but not $\delta=1.0=\eta$; the explanation may be in the high nonlinearity of the equations Eqns (9)-(11) implied by the choice $\delta=1.0=\eta$, this nonlinearity being reduced in the case $\delta=0.25=\eta$.

\section{Conclusion and perspectives}

In an effort to introduce the parallel in time discretization for the simulation of quantum 


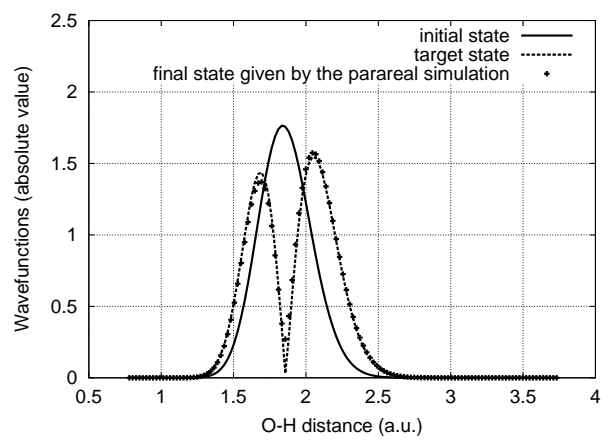

Figure 3: Plotting $\left|\psi_{0}\right|,\left|\psi_{T}\right|$ and $|\psi(x, T)|$ obtained with the controlled field. Complete overlap with the target is observed for the parareal scheme solved with the algorithm in Eqns (9)-(11) with $\delta=1=\eta$.

control problems we have presented here our preliminary studies that show the feasibility of the parareal implementations. Much work is still to be done in the direction of choosing more efficient coarse propagators and also in the adaptation of the overall scheme to equations (like those of the quantum control) where conservation laws are present. This results, even if encouraging, still need to be improved in order to gain in reliability and stability. The natural continuation would be the search for efficient entangled control-parareal formulation as in [5] in order to combine the parareal update directly with the control iterations.

Acknowledgments. It is a pleasure to acknowledge helpful discussions that we had on this topic with Prof. O. Pironneau ( JacquesLouis Lions Laboratory, Paris) and W. Zhu from Princeton Univeristy.

\section{References}

[1] A. Assion et al. Control of chemical reactions by feedback-optimized phase-shaped femtosecond laser pulses. Science, 282:919-922, 1998.

[2] L.Baffico, S.Bernard, Y.Maday, G.Turinici and G.Zerah Parallel in time molecular dynamics simulations, in preparation

[3] G. Bal and Y. Maday, "A "parareal" time discretization for the non-linear PDE's with application to the pricing of an American put", in Proceedings of the workshop on domain Decomposition, LNCSE Series, Springer Verlag, Zurich, 2001

[4] J.-L. Lions, Y. Maday and G. Turinici, $A$ "parareal" in time discretization of PDE's, C. R. Acad. Sci. Paris, Sér. I, Math. 332 (2001), no. $7,661-668$

[5] Y.Maday, G.Turinici A parareal in time procedure for the control of partial differential equations, C. R. Acad. Sci. Paris, Sér. I, Math. (2002), in press

[6] Y. Maday and G. Turinici New formulations of monotonically convergent quantum control algorithms, in preparation.

[7] S.Shi, A. Woody, and H.Rabitz, Optimal control of selective vibrational excitation in harmonic linear chain molecules, J.Chem Phys. 88(1988), p.6870

[8] W. Zhu, J. Botina and H. Rabitz Rapidly convergent iteration methods for quantum optimal control of population, J. Chem. Phys. 108(1998), 1953

[9] W. Zhu and H.Rabitz A rapid monitonically convergent iteration algorithm for quantum optimal control over the expectation value of a positive definite operator, J.Chem.Phys, 109 (2), p385-391, 1998 